\title{
The gut-liver axis as a target of liver disease management
}

\author{
Claudia Mandato ${ }^{1}$, Anna Pia Delli Bovi², Pietro Vajro ${ }^{2}$ \\ ${ }^{1}$ Department of Pediatrics, AORN "Santobono-Pausilipon", Naples, Italy; ${ }^{2}$ Pediatrics, Department of Medicine and Surgery University of Salerno, \\ Salerno, Italy \\ Correspondence to: Pietro Vajro, MD. Pediatrics, Department of Medicine and Surgery, University of Salerno, Via Allende, 84281 Baronissi, Salerno, \\ Italy. Email: pvajro@unisa.it. \\ Comment on: Albillos A, de Gottardi A, Rescigno M. The gut-liver axis in liver disease: Pathophysiological basis for therapy. J Hepatol 2020;72:558-77.
}

Submitted Mar 01, 2020. Accepted for publication Mar 18, 2020.

doi: 10.21037/hbsn.2020.03.27

View this article at: http://dx.doi.org/10.21037/hbsn.2020.03.27

The interplay between the gut and liver, the so called "gutliver axis" (GLA), plays a significant role in health and disease. As shown in Figure 1, the GLA is regulated by a number of equally relevant interacting factors including gut microbiota, integrity and function of the intestinal wall, antigen trafficking, mucosal innate immune response, and oxidative stress $(1,2)$. In addition to undigested or unabsorbed food and microorganisms, the gut lumen contains also their fermentation/metabolic products, bile, mucus, and epithelial cells spontaneously exfoliated from the gastrointestinal mucosa. The latter is composed of various cell types counting immune cells, goblet cells, and enterocytes strongly connected by tight junctions and covered with a mucus layer that acts as a dynamic barrier. Recent elucidation of a gut-vascular barrier (GVB) has revealed this structure to intervene to control gut bacteria and antigen translocation from the gut into the blood stream (3). Interestingly, diseases that are characterized or aggravated by impairment of the barrier function (so-called "leaky gut") and/or particular patterns of the microbiome (e.g., a lack of balance between Bacteroides and Firmicutes) are not restricted to the intestinal tract and liver. Some relevant examples are represented by diabetes type 1 , familial Mediterranean fever, obesity, cardiovascular disease, decreased bone-mass density, autism, dysregulation of immune response and systemic allergic diseases (1).

There is increasing evidence to suggest that any disturbance of the GLA (namely, dysbiosis and increased intestinal mucosa permeability/leaky gut) activates hepatic inflammation via toll-like receptor signaling in hepatocytes, induces oxidative stress, and may ultimately stimulate the progression from simple hepatic steatosis to non- alcoholic steatohepatitis (NASH). It has been proposed that the protective effects of the proper intestinal bacterial community may have beneficial effects for immunemediated liver injury (4). Interestingly, the GLA is disturbed also by endogenous alcohol produced by some bacteria in a similar manner to the effects of alcohol consumed in spirits. In recent years, the farnesoid X receptor (FXR), a nuclear transcriptional factor activated by bile acids that have been chemically modified by gut microbiota enzymes, has been indicated to have a role in maintaining the GLA properness, providing convincing proof of the central crosstalk between bile acids and gut microbiota. Bile acids acting through this dedicated receptor and $G$ protein-coupled bile acid receptor 1 (TGR5) in the ileum and liver may influence lipid metabolism, inflammation, and fibrogenesis (5).

There is evidence that a high-fat diet alters the microbiome, and that exercise and a healthy diet—such as the Mediterranean diet-contribute to a favorable regulation of the quantity and quality of microbiota (6). Thus, healthy lifestyle changes do represent an extraordinary ideal tool for the mitigation/prevention of NAFLD and the vast spectrum of other leaky-gut-related diseases as well. Unfortunately, patients are most often recalcitrant to these changes, meaning that their GLA remains constantly exposed to harmful factors. As there are no other currently effective treatments for non-alcoholic fatty liver disease (NAFLD) or alcohol-related liver disease, therapeutic targeting of the gut microbiota and bile acids in enterohepatic circulation appear promising approaches to cure these conditions (5).

Antibiotics and probiotics have been proposed for quite some time as predictably useful approaches to avert the intestinal dysbiotic consequences of obstinate sedentary 


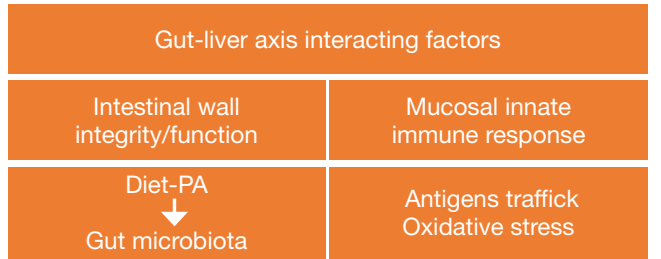

Figure 1 Interacting factors involved in the interplay between gut and liver. PA, physical activity.

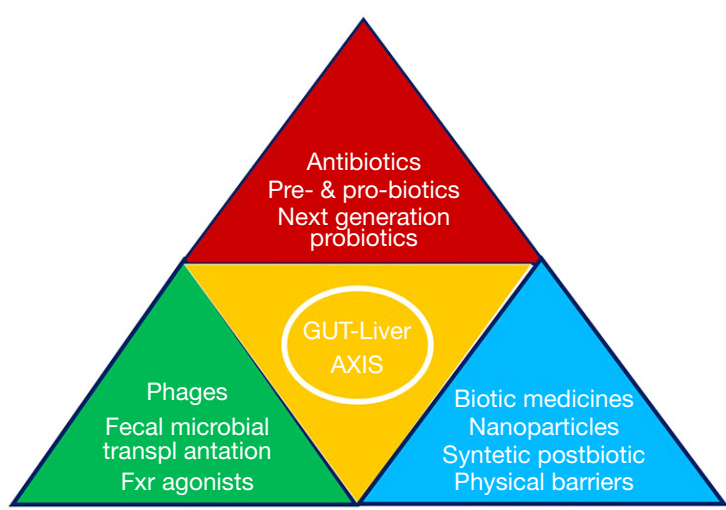

Figure 2 Synopsis of selected therapeutic interventions along the gut-liver axis.

lifestyle and Western diet $(7,8)$. While targeting the gut microbiota through antibiotics appears appropriate only in few conditions of proven small intestinal bacterial overgrowth, probiotic supplementation represents instead a tool with a wider field of application. Probiotics are defined as "live microorganisms which when administered in adequate amounts, confer a beneficial health effect on the bost". Given this definition, it is unsurprising that they may influence the resident gut microbiota profile and change the gut lumen to create a favorable environment with decreased production of reactive oxygen species and proinflammatory bacterial products involved in leaky-gut-related metabolic anomalies (9). Said that, unfortunately traditional probiotics used in NAFLD therapy have generally shown only some marginal ameliorative effects (10). Nonetheless, the gut microbiome will much probably remain an interesting candidate for a precision microbiomics $(11,12)$ due to an emerging class of next-generation probiotics (NGP). Studies aiming to elucidate the potential application of NGPs have discovered for instance that the imbalance between Bacteroides and Firmicutes affecting mucin glycosylation is counteracted by presence of the Akkermansia muciniphila, a key species particularly effective in increasing mucus thickness and increasing gut barrier function. This microorganism appears capable to lead to the reversal of obesity, insulin resistance, and other obesity-related conditions including $\operatorname{NAFLD}(7,8)$.

In an elegant seminal article, Albillos et al. recently summarized main advances on a wide array of most of these and other, either presently available or close to market, means able to modulate intestinal content, microbiome, mucus, and intestinal mucosa discussed during the Leuven 2018 Monothematic Conference of the European Association for the Study of the Liver (13). The authors have described how this novel armamentarium might repair the damage to the GLA caused by noxious attacks and prevent liver injury (Figure 2). Over and above customary probiotics, the article delineates the possible use of some other fascinating and entirely new tools, such as engineered probiotics that selectively consume toxic metabolites in the intestine, converting them into nontoxic forms and bioactive compounds (referred to as postbiotics). These metabolic products from intestinal bacteria include short-chain fatty acids, secondary bile acids, proteins, polysaccharides, vitamins, and organic acids, which act as metabolic regulators (14).

With respect to patients with cirrhosis, it is known that they exhibit marked impairment of the gut barrier paralleling the course of liver disease severity. Current medications, such as lactulose, proton-pump inhibitors, rifaximin, and other antibiotics, unfortunately can modify only in part the microbial functional and structural profile (15). Although the possibly appropriate and safe application of fecal or intestinal microbiota transplantation to liver disease requires further investigation, it appears to open up an exciting new area of research and therapy in the field of cirrhosis (15). Also obeticholic acid and other FXR agonists which target both the gut and liver are other tools currently being tested for efficacy not only in NAFLD but also in a number of other liver diseases, either stable or progressed to cirrhosis. In fact, by improving epithelial and vascular intestinal barrier functions these agents inhibit both pathogen-associated molecular patterns and bacterial translocation. Furthermore, they contribute to globally improve intestinal innate defense mechanisms, reduce intestinal inflammation, and decrease endotoxemia. But the story does not end here, for the effects of noxious intestinal contents may be counteracted also through a number of other novel strategies such as cellulose crosslinked with citric acid, insoluble crosslinked polymeric drugs, and carbon nanoparticles. Last but not least, bacteria targeting without inducing antibiotic resistance is now conceivable 
by means of phages engineered to specifically infect and kill intestinal bacterial pathogens (13).

In conclusion, GLA derangement plays an indisputable relevant role in several liver diseases, and a more and more wide array of tools presently available or close to market appears promising in its fine regulation. As recent evidence has revealed the microbiome to have a key role also in carcinogenesis, utilizing the gut microbiome as a diagnostic tool and therapeutic target might hopefully soon be beneficial for patients with hepatocellular carcinoma as well, particularly in terms of immunotherapy (16).

\section{Acknowledgments}

Funding: None.

\section{Footnote}

Provenance and Peer Review: This article was commissioned by the editorial office of Hepatobiliary Surgery and Nutrition. The article did not undergo external peer review.

Conflicts of Interest: All authors have completed the ICMJE uniform disclosure form (available at http://dx.doi. org/10.21037/hbsn.2020.03.27). The authors have no conflicts of interest to declare.

Ethical Statement: The authors are accountable for all aspects of the work in ensuring that questions related to the accuracy or integrity of any part of the work are appropriately investigated and resolved.

Open Access Statement: This is an Open Access article distributed in accordance with the Creative Commons Attribution-NonCommercial-NoDerivs 4.0 International License (CC BY-NC-ND 4.0), which permits the noncommercial replication and distribution of the article with the strict proviso that no changes or edits are made and the original work is properly cited (including links to both the formal publication through the relevant DOI and the license). See: https://creativecommons.org/licenses/by-nc-nd/4.0/.

\section{References}

1. Vajro P, Paolella G, Fasano A. Microbiota and gut-liver axis: their influences on obesity and obesity-related liver disease. J Pediatr Gastroenterol Nutr 2013;56:461-8.

2. Marciano F and Vajro P. Oxidative Stress and Gut Microbiota. In: Gastrointestinal Tissue. Chapter 8. Edited by Gracia-Sancho J and Salvado J (C 2017 Academic Press/ Elsevier Inc. London UK

3. Mouries J, Brescia P, Silvestri A, et al. Microbiota-driven gut vascular barrier disruption is a prerequisite for non-alcoholic steatohepatitis development. J Hepatol 2019;71:1216-28.

4. Abe K, Fujita M, Hayashi M, et al. Gut and oral microbiota in autoimmune liver disease. Fukushima J Med Sci 2020;65:71-5.

5. Arab JP, Arrese M, Shah VH. Gut microbiota in non-alcoholic fatty liver disease and alcohol-related liver disease: Current concepts and perspectives. Hepatol Res 2020;50:407-18.

6. Moosavian SP, Arab A, Paknahad Z. The effect of a Mediterranean diet on metabolic parameters in patients with non-alcoholic fatty liver disease: A systematic review of randomized controlled trials. Clin Nutr ESPEN 2020;35:40-6.

7. Chang CJ, Lin TL, Tsai YL, et al. Next generation probiotics in disease amelioration. J Food Drug Anal 2019;27:615-22.

8. Kim S, Lee Y, Kim Y, et al. Akkermansia muciniphila Prevents Fatty Liver Disease, Decreases Serum Triglycerides, and Maintains Gut Homeostasis. Appl Environ Microbiol 2020;86:e03004-19.

9. Mills S, Stanton C, Lane JA, et al. Precision Nutrition and the Microbiome, Part I: Current State of the Science. Nutrients 2019;11:923.

10. Vajro P, Mandato C, Veropalumbo C, et al. Probiotics: a possible role in treatment of adult and pediatric nonalcoholic fatty liver disease. Ann Hepatol 2013;12:161-3.

11. Paolella G, Mandato C, Pierri L, et al. Gut-liver axis and probiotics: their role in non-alcoholic fatty liver disease. World J Gastroenterol 2014;20:15518-31.

12. Cresci GAM, Lampe JW, Gibson G. Targeted Approaches for In Situ Gut Microbiome Manipulation. JPEN J Parenter Enteral Nutr 2020;44:581-8.

13. Albillos A, de Gottardi A, Rescigno M. The gut-liver axis in liver disease: Pathophysiological basis for therapy. J Hepatol 2020;72:558-77.

14. Gao J, Li Y, Wan Y, et al. A Novel Postbiotic From Lactobacillus rhamnosus GG With a Beneficial Effect on Intestinal Barrier Function. Front Microbiol 2019;10:477.

15. Bajaj JS, Khoruts A. Microbiota changes and Intestinal Microbiota Transplantation in Liver Diseases and Cirrhosis. J Hepatol 2020;72:1003-27.

16. Schwabe RF, Greten TF. Gut microbiome in HCCMechanisms, diagnosis and therapy. J Hepatol 2020;72:230-8.

Cite this article as: Mandato C, Delli Bovi AP, Vajro P. The gut-liver axis as a target of liver disease management. HepatoBiliary Surg Nutr 2021;10(1):100-102. doi: 10.21037/ hbsn.2020.03.27 